\title{
Debate sobre innovación institucional en el cooperativismo argentino
}

\author{
Carlos César Basañes \\ Gerente Confederación Cooperativa de la República Argentina Ltda. Cooperar \\ gerencia@cooperar.coop
}

Sumario: 1. Introducción.-2. Nuevos desafíos: 2.1. Plataformas cooperativas. 2.2. Cooperativas comunitarias para el desarrollo local. 2.3. Inclusión socio-laboral. 2.4. Cooperativas de pequeños productores familiares. 2.5. Cadenas de valor social y ambientalmente sostenibles. 2.6. Economía del cuidado. 2.7. Recuperación de empresas. 2.8. Medios de comunicación cooperativos.-3. Aspectos críticos. 3.1. El objeto de la cooperativa y el asociado colaborador. 3.2. Secciones de trabajo. 3.3. Gobernanza: distritos según tipo de vínculo asociativo.-4. Conclusión.-Referencias.

Resumen: En el marco de una legislación flexible, Argentina cuenta con un diversificado conjunto de modelos institucionales para la organización cooperativa. Sin embargo, se presentan situaciones donde se requiere innovar para viabilizar estratégicas asociativas en el campo de la economía digital, economía de cuidados, medios de comunicación y otros estrechamente vinculados al cumplimiento de los objetivos de desarrollo sostenible y a superar las fragilidades sociales que ha puesto en evidencia el Covid-19.

Frente a ello, en 2020, se comenzó a debatir sobre nuevas formas cooperativas, lo que dio lugar a dos nuevas resoluciones de la autoridad de aplicación. A partir de ello, se analizan los aspectos críticos y las respuestas encontradas para pensar modelos institucionales inspirados en la experiencia de las cooperativas de múltiples partes interesadas.

Palabras clave: cooperativismo, innovación, estatutos, actores múltiples.

Abstract: Within the framework of flexible legislation, Argentina has a diversified set of institutional models for cooperative organization. However, there are situations where innovation is required to enable associative strategies in the field of the digital economy, the care economy, the media and others closely linked to the fulfillment of the sustainable development objectives and to overcome the social fragility that it has put into evidence of Covid-19. 
Faced with this, in 2020, a debate began on new cooperative forms, which led to two new resolutions from the enforcement authority. Based on this, the critical aspects and the responses found are analyzed to think about institutional models inspired by the experience of multi-stakeholder cooperatives.

Keywords: cooperativism, innovation, bylaws, multi-stakeholder. 


\section{Introducción}

El cooperativismo argentino cuenta con una gran diversidad de modelos institucionales, adaptados a las distintas necesidades, en prácticamente todos los ámbitos de la actividad humana.

Esta diversidad no ha sido establecida por el marco normativo. La Ley 20.337 de Cooperativas (LC) no define ninguna tipología en particular, más allá de alguna referencia indirecta al establecer el tratamiento de los excedentes, y alguna breve mención de los bancos, cajas de crédito y cooperativas de trabajo. La diversidad es resultado de la práctica concreta de sus asociados que, a lo sumo, ha sido acompañada por la autoridad de aplicación al aprobar estatutos o reglamentos que presentan las propias cooperativas.

El cooperativismo agropecuario es quizás el que ha desarrollado mayor cantidad de innovaciones institucionales para adaptarse a las distintas actividades, dimensiones y objetivos. En dos trabajos realizados para el IICA ${ }^{1}$, hemos dado cuenta de la diversidad de modalidades adoptadas en temas como el tratamiento de capital (estas cooperativas han liderado la experiencia de sistemas de capitalización proporcionales y fondos rotativos de capitalización), sistemas de participación (incluyendo el desarrollo de sofisticados diseños institucionales para dar respuesta a la dispersión territorial y a la diversidad de servicios), múltiples formas de organización del trabajo, distintas modalidades de integración a la cadena de valor y al territorio, etc.

El cooperativismo de servicios públicos, próximo a cumplir cien años, también ha desarrollado modelos institucionales muy valiosos, en el marco de una relación con el poder público concedente tan innovadora como compleja. Resulta especialmente significativa su gradual transformación desde un rol inicial de proveedores de un servicio público específico hacia un papel de verdaderas agencias comunitarias de desarrollo local, que incluyen un menú muy diversificado de servicios dirigidos a las familias y a las empresas (Suárez Area, 2018).

Especialmente significativo ha sido el esfuerzo que han requerido para incorporar los servicios vinculados a las nuevas tecnologías de la información y comunicación. Ello incluye modalidades innovadoras de asociación entre cooperativas para el desarrollo de servicios de internet y servicios de cuatro play, incluyendo la gestión conjunta de licencias de operadores móviles virtuales.

1 Ver (Basañes, Obschatko, Martini; 2011) y (Basañes, Amantini; 2017). 
El cooperativismo de trabajo ha adquirido especial dimensión en las primeras décadas del siglo XXI, con experiencias muy diversas, desde cooperativas industriales emergentes de procesos de recuperación de empresas, hasta cooperativas de cuidadores que están naciendo de la mano de los nuevos paradigmas de la llamada economía del cuidado; desde las cooperativas emergentes de planes públicos de inserción socio laboral, hasta cooperativas de programadores que están logrando una poderosa simbiosis entre software libre y autogestión.

En la actualidad, gran parte del debate sobre modelos de organización y sobre las normas que requieren, se registra, precisamente, en el ámbito del cooperativismo de trabajo. Ello es producto de la centralidad que estas cooperativas tienen en la creación de puestos de trabajo - en el contexto de las severas restricciones que presenta la economía argentina para crear trabajo asalariado aún en condiciones de crecimiento- - y de la centralidad que también tienen en la búsqueda de nuevas formas de organización ante el impacto de las nuevas tecnologías².

Un rápido repaso sobre la diversidad de modelos que encontramos en Argentina, debe incluir también a las cooperativas de vivienda (con o sin trabajo autogestionado, y con diversidad de modelos de financiamiento), cooperativas de consumo (desde pequeñas iniciativas locales vinculadas al compre ecológico, hasta cadenas de supermercados), escuelas cooperativas (incluyendo aquellas gestionadas por los trabajadores y aquellas gestionadas por madres y padres), cooperativas de medios de comunicación (radios, televisión, periódicos), cooperativas de servicios para distintos tipos de pequeñas y medianas empresas (almacenes, supermercados familiares, carpinterías, pescadores, etc., destacándose muy particularmente, por su dimensión, las cooperativas de farmacéuticos), cooperativas de provisión de servicios de salud, banco y cooperativas de crédito, aseguradoras, etc.

La ley de cooperativas ha demostrado ser un marco suficientemente flexible para permitir el desarrollo de toda esta diversidad. Esta flexibilidad, por ejemplo, se ha visto reflejada en las distintas modalidades que han adoptado las cooperativas argentinas para implementar sus estrategias asociativas, en el marco del principio de cooperación entre cooperativas.

En el año 2014, el Gobierno Nacional puso en debate un proyecto de «Ley Federal de Cooperativas y Mutuales» que incluía una fuerte

2 Al respecto ver, por ejemplo, el capítulo «El camino de la cooperación hacia el futuro del Trabajo» en (Guarco, 2020). 
modificación del marco legal para la integración. Allí se proponía una estructura de uniones locales (de nivel municipal), federaciones (provinciales o por rama) y confederaciones de carácter nacional.

Este planteo no tuvo consenso. Desde el movimiento cooperativo se opinó que no parecía razonable pasar del modelo altamente diversificado y flexible de integración cooperativa en Argentina, a un esquema fuertemente estructurado y jerarquizado como el que se proponía.

En aquella oportunidad, como contribución al debate, preparamos un documento de trabajo (Basañes, 2015) que incluía un inventario de modelos de integración adoptados en Argentina, con el propósito de evidenciar una diversidad que no podía encorsetarse en una legislación como la que se proponía. Por ser representativo de la flexibilidad institucional del cooperativismo en Argentina, se reproducen aquí las experiencias más significativas que se señalaban en dicho documento:

- Cooperativas de membresía mixta. Como por art. 17 de la LC los asociados pueden ser de cualquier personería, existen importantes experiencias donde cooperativas y otros tipos de empresas se articulan con objetivos comunes. Es, por ejemplo, la experiencia de DYPRA, cooperativa integrada por editoriales de diarios y revistas de todo el país, algunas de ellas cooperativas y otras de distinta personería jurídica. También es el caso de COLSECOR, integrada por empresas que gestionan servicios de televisión por cable bajo distintas formas jurídicas.

- Federaciones empresarias sectoriales. Entre las cooperativas agropecuarias existen numerosas experiencias de integración cooperativa para que el productor avance en el control de la cadena de valor (comercio mayorista, exportación, industrialización, etc.). Habitualmente organizadas como federaciones, suelen contar con sistemas de representación proporcional; algunos de ellos muy innovadores, como por ejemplo el de la Asociación de Cooperativas Argentinas, donde tiene más votos quien es más consecuente (mayor razón entre el volumen que comercializa con ACA y el total), más allá de su tamaño.

- Federaciones territoriales multisectoriales. Existen cooperativas representativas de alguna región, o de una provincia, que desarrollan tanto actividad gremial como servicios económicos a sus asociadas. Ejemplos son la Federación de Cooperativas de La Rioja, la Federación de Cooperativas de Corrientes, o la Asociación Intercooperativa Regional, del sur de la Provincia de Buenos Aires. 
- Federaciones territoriales de un sector específico. Un ejemplo es la antigua Federación de Cooperativas Agropecuarias de Misiones, que ha tenido etapas de actividad económica (administrando un molino yerbatero) y etapas de actividad estrictamente gremial. Este ejemplo evidencia por la imposibilidad de discriminar taxativamente entre cooperativas de representación y cooperativas con actividad económica, como se pretendía en el proyecto legislativo.

- Federaciones nacionales de carácter sectorial y confederaciones nacionales. A diferencia de lo que proponía el proyecto, existen experiencias de federaciones sectoriales de carácter nacional como la Federación Argentina de Cooperativas de Consumo, la Federación Argentina de Cooperativas de Telecomunicaciones de la República Argentina o la Federación Argentina de Cooperativas Eléctricas. En este último caso, algunas de sus asociadas integran a su vez alguna federación provincial. Complementariamente también existen estructuras confederativas sectoriales como la CONAICE (que agrupa a federaciones provinciales de electricidad, en un modelo alternativo al de FACE), la decana Confederación Intercooperativa Agropecuaria (Coninagro), o las más recientes confederaciones de trabajo (CNCT y Conarcoop), que incluyen tanto federaciones de carácter gremial (nacionales y provinciales) como entidades de carácter económico (red gráfica, red textil cooperativa). Finalmente, esta diversidad se completa con COOPERAR, organización confederativa nacional que integra cooperativas de todo tipo.

- Redes de integración gremial y económica. Las cooperativas de servicios públicos suelen tener dobles o triples asociaciones dependiendo sus necesidades. Por ejemplo, están asociadas a alguna federación de perfil gremial como la Federación de Cooperativas de Servicios Eléctricos de la Provincia de Buenos Aires, mientras que algunas además se asocian a cooperativas para determinados servicios, como NODOSUD (internet), TRAMA (producción de contenidos audiovisuales), o «La Regional» (compras conjuntas de vehículos e insumos), también en la provincia de Buenos Aires. Todas estas instancias de integración configuran una red flexible que busca adecuarse a las necesidades de cada territorio y actividad.

- Asociaciones civiles. Existen cooperativas que se integran a través de una asociación civil, integración que puede o no contar con otras figuras jurídicas. Por ejemplo, la Asociación de Prestadores Eléctricos de la Provincia de Buenos Aires (APEBA) y 
la Cámara de Empresas Líderes de Seguridad e Investigaciones (CELSI).

- Cooperativas de primer grado referenciadas en cooperativas de base. Hay cooperativas, como La Segunda (seguros), que si bien son cooperativas de primer grado su consejo está integrado por representantes de cooperativas de primer grado, dado que las mismas funcionan como sus distritos electorales.

- Sociedades instrumentales. Existen muchas cooperativas que descentralizan actividades y buscan capital de riesgo a partir de sociedades comerciales con participación cooperativa. En algunos casos estas sociedades resultan sistemas de integración intercooperativa, como el caso de Sodecar, que se conformó como instancia de integración de Uncoga (federación de cooperativas de ganadería) y Sancor (industria láctea).

- Megacooperativas. Otros esquemas de integración han apelado a las fusiones y absorciones de cooperativas, desarrollando complejos sistemas de participación que dan cuenta de las organizaciones territoriales preexistentes. Es el caso del Banco Credicoop, resultante de la fusión de cajas y bancos cooperativos, y de Agricultores Federados Argentinos, resultante de la fusión y absorción de 26 cooperativas agropecuarias.

- Grupos cooperativos. Existen grupos empresarios controlados por una misma conducción, pero articulados a través de múltiples empresas como el Grupo ACA, Sancor Seguros, etc.

- Agrupaciones de colaboración cooperativa. A partir de la herramienta de las agrupaciones de colaboración empresaria, se desarrollan emprendimientos conjuntos entre cooperativas o entre cooperativas y otros tipos de empresas. Ejemplo del primer caso es la agrupación que forman la Cooperativa Obrera (consumo) con la Cooperativa Agropecuaria de Sombra del Toro, para la gestión asociativa de un frigorífico.

- Redes de cooperativas de trabajo. Bajo la forma de federaciones o de cooperativas de cooperativas, existen redes de cooperativas de trabajo donde se articulan los procesos de trabajo de las distintas organizaciones de base.

- Federaciones de promoción cooperativa. Es el caso del Instituto Movilizador de Fondos Cooperativos, que nace como una entidad de integración económica entre cajas de crédito, y deviene en una federación de cooperativas de todo tipo, con objetivos de promoción, capacitación, difusión cultural, etc. Es una organización de carácter nacional, no está asociada a ningún sector o territorio en particular, y desarrolla tanto actividades de 
tipo gremial cooperativo como de tipo económico (editorial, actividades culturales, organización de una universidad, microcrédito, etc.).

Todas estas experiencias configuran un denso entramado de relaciones asociativas, que no responden a un esquema meramente piramidal, y que no son resultado del diseño de ninguna norma. Son las formas que ha adoptado el movimiento cooperativo en forma autónoma, en el marco flexible de la LC.

\section{Nuevos desafíos}

A partir de esta experiencia caracterizada por la diversidad y la flexibilidad, el cooperativismo en Argentina enfrenta nuevos desafíos para los que existen limitaciones dentro de los modelos adoptados hasta ahora. Ello ha abierto un debate, particularmente intenso desde 2020, del cual daremos cuenta en lo que sigue.

En primer lugar, revisemos cuáles serían las situaciones donde los modelos conocidos resultan insuficientes.

\subsection{Plataformas cooperativas}

La expansión de la llamada economía de plataforma está teniendo un fuerte impacto en la reorganización de sectores como logística, finanzas, transporte, etc. El cooperativismo está explorando la forma de desarrollarse en este ámbito. Algunos trabajos que reflejan ello son (Mayo, 2019), (Vanini, 2019) y (Guarco, 2020: 148).

Estas iniciativas parten en general de una mirada crítica sobre el modelo empresarial que hoy hegemoniza la economía de plataforma, que se fundamenta al menos en tres argumentos: a) La posición dominante sobre sus usuarios que tiene las empresas multinacionales que controlan estas plataformas, que se refleja en precarización del trabajo y en el cobro de comisiones abusivas a las familias o a las pequeñas empresas adheridas, b) la pérdida de divisas que implica la transferencia de beneficios al exterior para el desarrollo de actividades que se limitan a vincular a actores locales, y c) la ruptura de lazos sociales entre actores locales, cuyas relaciones son mediatizadas por empresas sin arraigo ni compromiso con el desarrollo local.

En Argentina existen varias iniciativas de cooperativas en este campo. Por el ejemplo, las impulsadas por la Federación Argentina de 
Cooperativas de Trabajo de Tecnología, Innovación y Comunicación (Facttic). Esta organización, integrada por 28 cooperativas de programadores, es quizás la experiencia más innovadora en el ámbito del cooperativismo de trabajo. Es resultado del esfuerzo asociado de jóvenes profesionales de la industria del software que buscaron desarrollarse en un ámbito democrático y participativo, y que sostienen principios de soberanía tecnológica, soberanía de datos, alfabetización digital ciudadana y democratización del mercado de la tecnología (Cooperar, 2019: 66).

Varias de sus asociadas están trabajando en el desarrollo de plataformas de base cooperativa, en contacto, por ejemplo, con la experiencia de la Federación Coopcycle, con presencia en países de Europa y Norteamérica.

Al momento de querer desarrollar estas iniciativas, habitualmente en ciudades de tamaño medio, es necesario desarrollar instancias colaborativas entre múltiples actores, por ejemplo: a) desarrolladores de la plataforma, que eventualmente la gestionarán, b) trabajadores del servicio de reparto, c) pequeños y medianos comercios de la localidad que ofrecen sus servicios en la plataforma, d) compradores y usuarios de los servicios (familias) y e) el municipio y otras instituciones de la localidad, interesados en desarrollar un servicio que fortalezca las cadenas de valor de carácter local.

En este punto, es donde los modelos institucionales existentes en Argentina quedan estrechos. Suele apelarse al modelo de la cooperativa de trabajo - por ser el de mayor flexibilidad para acordar las condiciones de trabajo- pero este deja afuera a actores que pueden aportar recursos, liderazgo institucional y representación de otras necesidades que la plataforma debe satisfacer, más allá de la generación de trabajo decente.

\subsection{Cooperativas comunitarias para el desarrollo local}

En pequeñas localidades de Argentina existen cooperativas constituidas por su comunidad, orientadas a generar trabajo y así evitar el éxodo de sus habitantes, muy particularmente de la juventud. Se trata de cooperativas donde los asociados son miembros de toda la comunidad, y el trabajo está organizado en forma asalariada, esto es, los trabajadores son contratados en el marco de la ley de contrato de trabajo y de los convenios colectivos que correspondan a la rama de actividad.

Habitualmente, desde lo formal, son cooperativas de consumidores y/o de productores, pero ello no se ajusta estrictamente a la realidad. La mayor parte de su actividad no está vinculada a comercializar o 
industrializar la producción de sus asociados agricultores, ni a proveer bienes o servicios a sus asociados. El objetivo es crear trabajo, y así beneficiar indirectamente a toda la comunidad. No brindar un servicio o bien específico a sus asociados.

Este es un fenómeno que aún no ha sido receptado por la autoridad de aplicación. Ni en su tarea de registro (no existen modelos de estatuto que contemplen esta modalidad), ni en sus acciones de promoción.

Vinculado a este concepto, en 2020, algunos municipios impulsaron el debate sobre la necesidad de un nuevo tipo de cooperativas, que provisoriamente bautizaron como cooperativas de "desarrollo local». Se exploró la posibilidad de constituir cooperativas cuyo objetivo fuese crear puestos de trabajo en sus respectivos municipios, que estuviesen fundadas y gobernadas por distintas instituciones locales, como por ejemplo el Estado municipal, cooperativas de servicios públicos, asociaciones comerciales y otras organizaciones no gubernamentales.

Serían cooperativas comunitarias, en los términos que aquí hemos definidos, pero donde la representación de la comunidad estaría mediatizada por distintas organizaciones gubernamentales y no gubernamentales. Este modelo continúa en debate.

\subsection{Inclusión socio-laboral}

Se trata de cooperativas cuyo objetivo es la inclusión socio laboral de personas con algún tipo de desventaja, en particular con consumos problemáticos o usuarias de servicios de salud mental. Son experiencias emparentadas con las cooperativas sociales italianas de tipo B (Ley italiana 381 de 2009). De hecho, algunos de sus impulsores han tenido un estrecho contacto con el movimiento cooperativo italiano.

Todas están organizadas como cooperativas de trabajo, lo que no refleja adecuadamente la diversidad de sujetos que efectivamente las gestionan: personas que adolecen de alguna desventaja, trabajadores que operan como acompañantes y que hacen viable la organización, voluntarios vinculados a distintas instituciones (asociaciones barriales, iglesias, etc.) y en ocasiones profesionales de servicios públicos, sociales o de salud.

La limitación de la cooperativa de trabajo para dar cuenta de esta diversidad de actores, ha sido especialmente conflictiva en cooperativas vinculadas a la salud mental. En estos casos resulta imprescindible construir una gobernanza que facilite la participación de los trabajadores, sus familias y otros voluntarios, y que ello además pueda realizarse en forma articulada con las prácticas terapéuticas que son responsabilidad del servicio de salud. 
Es importante señalar que Argentina cuenta con una ley de salud mental en muchos aspectos de vanguardia (Ley 26.657 de 2011), que persigue la desmanicomialización y que ordena a las autoridades sanitarias coordinar con las áreas de educación, de desarrollo social, de trabajo y otras, acciones de inclusión social, laboral y de atención en salud mental, de carácter comunitario, por ejemplo, a través de cooperativas de trabajo (art. 11).

Viabilizar esto, requiere modelos institucionales adecuados, que aún no están debidamente desarrollados.

\subsection{Cooperativas de pequeños agricultores familiares}

En Argentina, el cooperativismo agropecuario, en consonancia con la mayor parte de la experiencia internacional, se ha desarrollado en base a cooperativas donde sus asociados son productores que reciben distintos servicios (comercialización, industrialización, provisión de insumos, asistencia técnica, etc.), y para cuya ejecución contratan trabajadores en el marco de los respectivos convenios colectivos (más allá de la existencia de alguna contribución de trabajo voluntario aportado por sus asociados, propia de las relaciones de reciprocidad que caracterizan a este tipo de entidades).

A este modelo institucional prevaleciente, se suma alguna pequeña y aislada experiencia de cooperativas de trabajo, donde los asociados explotan en forma colectiva un predio rural bajo distintas formas de tenencia de la propiedad o desarrollan alguna actividad agroindustrial.

Estos modelos institucionales han mostrado limitaciones para materializar las estrategias asociativas que requieren los productores más pequeños, en situación de pobreza. Cuando desde el Ministerio de Agricultura, Ganadería y Pesca, junto con el IICA, se investigó a las cooperativas en el ámbito rural, se concluyó que el estrato con menor presencia de cooperativas era el de los productores familiares más pequeños. Incluso se registró una enorme cantidad de fracasos entre las cooperativas que se habían constituido luego de la crisis económica del 2001 para dar respuestas a esos pequeños productores.

Al indagar sobre las razones de este fracaso, uno de los temas identificados fue la falta de modelos institucionales adecuados para dar cuenta de las necesidades de este tipo de productor: "Cualquiera de los modelos de estatuto hoy vigentes obliga a diferenciar el carácter de productor y el de trabajador, roles que en la agricultura familiar se confunden, en particular cuando se trata de los productores más pequeños. Las estrategias asociativas de éstos están vinculadas a mejorar 
la retribución a su trabajo (y a mejorar el resto de las condiciones y medio ambiente de trabajo) independientemente de si el trabajo se realiza en sus pequeños predios particulares, en predios de otros asociados en el marco de una estrategia asociativa, en pequeños emprendimientos asociativos de carácter agroindustrial, u ofreciendo en forma asociada su trabajo» (Obschatko, Basañes, Martini, 2011: 145).

La situación más evidente se presenta cuando un grupo de pequeños productores desean impulsar una pequeña actividad agroindustrial que agregue valor a su producción y que cree puestos de trabajo para sus familias. La nueva actividad habitualmente no es capaz de generar salarios como los exigidos por las condiciones de los convenios colectivos que, por otro lado, están muy alejados del nivel de ingreso que obtienen las familias por su trabajo rural.

En la publicación citada no se postula que debe haber un modelo específico para pequeños productores, sino que deben existir distintas posibilidades según las diferentes estrategias que pueden adoptar los asociados. Por ejemplo, cooperativas agrarias de gestión de la producción, cooperativas de agroindustria familiar, cooperativa de trabajo de producción colectiva, cooperativas de trabajo para servicios rurales, y la combinación de cada uno de estos modelos teóricos (Obschatko, Basañes, Martini; 2011: 49 y 140).

En (Basañes, Amantini; 2017: 66) hemos estudiado la experiencia de la Federación de Cooperativas Agropecuarias de la Provincia de San Juan (Fecoagro), que está integrada por cooperativas agropecuarias y por cooperativas de trabajo, pero que al analizarse detalladamente cada una de ellas, la mayoría respondía a un formato híbrido que no se ajusta cabalmente a ninguno de los modelos formalmente establecidos en sus estatutos sociales.

\subsection{Cadenas de valor social y ambientalmente sostenibles}

El cumplimiento de los objetivos de desarrollo sostenible exige una profunda transformación en las formas de producción y de consumo de alimentos, lo que solo será posible con el esfuerzo articulado de toda la cadena de valor.

Este debate está presente en el movimiento cooperativo global. Por ejemplo, la Alianza Cooperativa Internacional (ACI), al momento de escribir estas líneas, está convocando a su Congreso Mundial Cooperativo (Seúl, diciembre de 2021). Allí, en el eje de seguridad alimentaria, propone debatir lo siguiente: «La relación entre la producción alimentaria y el consumo ha sido probada desde diferentes prismas: cambio 
climático, sequía, inundaciones, desplazamientos de refugiados o despoblación en las regiones rurales. Las cadenas de producción y distribución se han visto alteradas. No obstante, esto ha permitido renovar el papel de las cooperativas comprometidas con la producción agrícola. ¿Cómo pueden encontrar las cooperativas nuevas estrategias para asegurar la producción en los sistemas alimentarios? ¿Cómo contribuye eso a construir cadenas de valor sostenibles, éticas e inclusivas?» (https://icaworldcoopcongress.coop/).

Las cooperativas más interpeladas por este planteo son las cooperativas de consumo - que podrían canalizar el compromiso de consumo social y ambientalmente responsable de sus asociados- y las cooperativas agropecuarias — que podrían materializar el compromiso de sus productores asociados con la sostenibilidad su actividad productivaPor supuesto, esto adquiere mayor potencial si se logran estrategias compartidas de cooperativas de consumo y agropecuarias, tanto en el ámbito de cada economía nacional como en el ámbito internacional. Esa es la gran apuesta de la $\mathrm{ACl}$ : la construcción de cadenas de valor éticas globales, articuladas desde el movimiento cooperativo.

En Argentina, la experiencia más avanzada en este sentido, es la Central de Compra Virtual que administra la Federación Argentina de Cooperativas de Consumo (FACC), a través de la cual organiza la compra asociada de sus integrantes, priorizando la adquisición a cooperativas de producción (agropecuarias o de trabajo) ${ }^{3}$. En la actualidad FACC está explorando articular esta iniciativa con el cooperativismo de consumo de Uruguay y Brasil.

Convergente con estos objetivos, han surgido múltiples iniciativas que procuran un diálogo directo entre consumidores, habitualmente urbanos, y productores familiares vinculados a la producción agroecológica o en tránsito hacia la agroecología.

Los formatos asociativos que adquieren estas iniciativas son muy variados, y a menudo informales. En Argentina ha sido especialmente relevante el surgimiento de "cooperativas comercializadoras». Se trata de cooperativas de trabajo que brindan servicios logísticos de distribución de alimentos, que en general operan articulando la oferta de pequeños productores familiares y de cooperativas agroindustriales de producción de alimentos (dulces, vinos, yerba mate, azúcar, pastas secas, arroz, etc.), con la demanda de organizaciones sociales vinculadas al consumo responsable o al consumo popular, y complementariamente a través de la venta minorista en locales propios («almacenes cooperati-

3 https://www.faccargentina.coop/ 
vos») y/o venta a pequeños comercios del ramo (generalmente identificados como «dietéticas») 4 .

Este tipo de «comercializadoras» son la respuesta que encontraron organizaciones de productores y organizaciones sociales que representan a familias consumidores, para resolver el problema logístico de la distribución.

Estas experiencias, junto con otras como los mercados comunitarios 0 «mercados de la economía social» ${ }^{5}$, y los mercados frutihortícolas organizados como cooperativas $u$ otras formas asociativas ${ }^{6}$, son antecedentes que nos invitan a pensar nuevas formas de cooperativas, donde puedan institucionalizarse los esfuerzos colaborativos de los distintos actores comprometidos en la construcción de cadenas agroalimentarias ambiental y socialmente sostenibles: los consumidores y las distintas organizaciones sociales preocupadas por el consumo responsable, productores agropecuarios, cooperativas de trabajo agroalimentarias, municipios, etc.

\subsection{Economía del cuidado}

En la última década han comenzado a surgir cooperativas de cuidados personales, orientadas a brindar servicios de cuidados a personas que sufren algún tipo de desventaja que limita su autonomía, ya sea por su edad (adultos mayores y niños) o por enfermedades o discapacidades. Están inspiradas en las cooperativas sociales italianas tipo A (Ley italiana 381 de 2009). Algunos de sus impulsores en Argentina han tenido estrecho contacto con el movimiento cooperativo italiano.

Desde las políticas públicas se considera que este tipo de cooperativas puede ser de gran impacto para la rápida generación de puestos de trabajo, al tiempo que se fortalecen los sistemas de cuidados personales, cuyas debilidades han quedado evidenciadas durante la pandemia del Co-

4 Un ejemplo es la experiencia de Alimentos Cooperativos, iniciativa que surgió desde la Comisión de Economía Regional de Cooperar, a partir de la iniciativa de federaciones de cooperativas de productores de alimentos y de cooperativas de trabajo. https://alimentoscooperativos.com/

5 Por ejemplo, los Mercados Productivos Asociativos de la Provincia de Río Negro (Ley provincial 4439), comentados en (Gallo, Grillo, 2011) o las distintas experiencias presentadas el Eje "Mercados, comercialización, consumo y finanzas, del II Congreso Nacional de la Economía Social y Solidaria, organizado por universidades nacionales integrantes de la Red Universitaria de Economía Social y Solidaria, auspiciado por Cooperar. (Errecalde, 2020)

6 Como el Mercado Cooperativo de Guaymallén, provincia de Mendoza, o la Sociedad de Chacareros Temporarios, provincia de San Juan (Basañes, Amantini, 2017: 74) 
vid-19. Por el ejemplo, el trabajo de cuidado es una de las cuatro líneas de actividad priorizadas por el Programa Potenciar Trabajo, que es el principal programa del Ministerio de Desarrollo Social para contribuir a la inserción socio laboral de personas en situación de vulnerabilidad social.

Este tipo de cooperativas tienen también un fuerte vínculo con las políticas de igualdad de género, a partir del diagnóstico de que gran parte de las desventajas que sufren las mujeres se explican por la desigual distribución del trabajo de cuidado (Guarco, 2020: 41). Por ello, el recientemente creado Ministerio de Mujeres, Diversidad y Género se encuentra abocado al diseño de una ley que cree un sistema nacional de cuidados, proyecto que se está debatiendo en una comisión de la que participa el movimiento cooperativo.

En Argentina las cooperativas de cuidado se organizan como cooperativas de trabajo. Nuevamente, cabe preguntarse, si no es posible explorar modelos que permitan la participación de otros actores, como los usuarios de los servicios. De hecho, en la promoción de este tipo de cooperativas, han participado cooperativas de servicios públicos y autoridades locales (de alguna manera representando el interés de la comunidad de contar con estos servicios). La Universidad Nacional de Tres de Febrero, ha realizado un detallado seguimiento de todo este proceso, que puede consultarse en (Bragulat, 2020).

\subsection{Recuperación de empresas}

La recuperación de empresas a través de la organización cooperativa ha sido un fenómeno muy importante en Argentina, desde la crisis de 2001. La casi absoluta mayoría de estas cooperativas se organizaron como cooperativas de trabajo, y su constitución fue mayoritariamente resultante de la resistencia de los trabajadores ante el riesgo de perder su empleo en el marco de un proceso de quiebra.

Dada la crisis que sufren las pequeñas y medianas empresas en Argentina, luego de tres años de caída del producto bruto interno (2018 a 2020), resulta aconsejable pensar en modelos alternativos para la recuperación de empresas.

En los años recientes, algunos supermercados de propiedad familiar han sido absorbidos por la Cooperativa Obrera Ltda7. Estos casos fueron resultado de la puesta en contacto de esta cooperativa de con-

7 Es la mayor cooperativa de consumo en argentina. Tiene más de dos millones de asociados y es propietaria de supermercados en más de 70 ciudades de cinco provincias argentinas. https://www.cooperativaobrera.coop/ 
sumo con los trabajadores y con los propietarios originales que deseaban abandonar la actividad, a los efectos de preservar los puestos de trabajo. Todo ello acompañado por el gobierno municipal, que también necesitaba evitar el conflicto social que hubiese derivado del cierre de la empresa.

Sin embargo, estos procesos no son visualizados por la academia a la hora de estudiar la recuperación de empresas, ni son objeto de políticas especifica o proyectos de leyes, como los que hoy se están debatiendo en el Congreso Nacional para favorecer la sostenibilidad de las cooperativas de trabajo emergentes de procesos de recuperación de empresas.

Una política del gobierno o del movimiento cooperativo dirigida a facilitar la cooperativización de empresas en crisis, debe incluir financiamiento, asistencia técnica y modelos institucionales adecuados y flexibles para viabilizar distintas estrategias asociativas.

Esto último nos remite a las «cooperativas comunitarias en el desarrollo local» que presentamos en 2.2, o a otros modelos de cooperativas donde puedan articularse los esfuerzos de consumidores, trabajadores, productores o simplemente miembros de la comunidad a los que les interese apoyar la recuperación de la empresa en un marco de gestión democrática y participativa.

Este debate es tan incipiente como necesario en Argentina. Durante el 2020, desde el gobierno se promovieron reuniones a distancia con el cooperativismo de Quebec (Canadá) para conocer su experiencia en la organización de cooperativas de múltiples partes interesadas para la recuperación de empresas.

\subsection{Medios de comunicación cooperativos}

En Argentina se ha desarrollado, en las últimas décadas, una importante experiencia de cooperativas en el ámbito de los medios de comunicación. Incluye prensa gráfica, radios y televisión. Ha sido, por ejemplo, significativo el avance de las cooperativas de servicios públicos en la provisión de servicios de televisión por cable (tardíamente autorizado por la ley 26.522 de 2009; antes estaba prohibido por un decreto-ley de la última dictadura). Estas cooperativas no solo brindan el servicio, sino que además desarrollan contenidos propios, procurando reflejar las voces de la comunidad (Guarco, 2020: 79).

Periódicos y portales de noticias gestionados por sus trabajadores, y radios comunitarias pertenecientes a cooperativas o mutuales, forman parte de este muy diverso mundo de los medios de comunicación pertenecientes a empresas de la economía social. 
Todos ellos enfrentan la fuerte reconfiguración de los medios de comunicación provocada por las nuevas tecnologías de la información y la comunicación (crisis de la prensa gráfica, avance de las plataformas sobre la televisión abierta, y un largo etcétera) y la concentración económica en esta actividad. Frente a ello las cooperativas, y en general todas las pequeñas y medianas empresas de medios de comunicación, tienen la necesidad de desarrollar esfuerzos colaborativos con distintos actores de la comunidad.

Esto es muy evidente en el caso de los periódicos en crisis recuperados por sus trabajadores, donde su sostenibilidad requiere el compromiso de estos trabajadores, pero también de sus lectores. Un modelo institucional que permitiese la participación de estos dos actores resultaría muy potente, dado que permitiría tanto la defensa del derecho de los trabajadores, como la democratización de la línea editorial del medio, a partir de la participación de los periodistas y de los usuarios de servicio periodístico.

\section{Aspectos críticos para avanzar en nuevos modelos institucionales}

En el contexto del inicio de una nueva gestión del Gobierno Nacional, en 2020 avanzó el debate sobre la necesidad de innovar en materia de modelos de cooperativas. Ello se dio en el marco del Consejo Consultivo y de la Comisión Técnica Asesora en Desarrollo Local del Inaes 8 , bajo el título de "cooperativas de múltiples partes interesadas» o de "cooperativas de desarrollo local».

Como un primer resultado de dicho proceso, el Directorio del Inaes, mediante Resolución 478/2020 de "Cooperativas de Actores Múltiples», reafirmó que «existe la posibilidad de constituir entidades cooperativas cuyos asociados sean actores que cumplen diferentes roles en la cadena de producción y consumo» y destacó, en sus fundamentos, la posibilidad legal de aceptar la multiplicidad de objetos sociales y de

8 El Instituto Nacional de Asociativismo y Economía Social (Inaes) es la autoridad de aplicación de la LC y de la ley de mutualidades (Ley 20.321). Esta conducido por un Directorio integrado por tres representantes del Poder Ejecutivo, dos del movimiento cooperativo y dos de las mutuales. En el 2019 se constituyó un Consejo Consultivo con el fin de asesorar el Directorio, con la participación de referentes del ámbito académico y de las organizaciones del sector. Por otro lado, en el 2020, se conformaron Comisiones Técnicas Asesoras, con profesionales del sector y funcionarios del Inaes, para contribuir al diseño de las políticas de promoción en los distintos ámbitos. 
asociar personas de distintos carácter jurídico tanto a las cooperativas de primer grado como a las cooperativas de grado superior.

Más allá de su carácter limitadamente declarativo, esta Resolución expresa la voluntad política, acordada por los representantes de movimiento cooperativo y del Poder Ejecutivo que integran el Directorio, de avanzar en modelos institucionales innovadores mediante la aprobación de estatutos sociales y reglamentos que reflejen la existencia de múltiples partes interesadas.

Siguiendo este camino, se avanzó, en el marco de la Comisión de Normativa de Cooperar ${ }^{9}$, del Consejo Consultivo y de la Comisión Técnica Asesora de Desarrollo Local del Inaes, en el diseño de algunos proyectos de estatutos innovadores, en diálogo con los municipios que deseaban impulsar cooperativas de desarrollo local, y con cooperativas de inclusión socio laboral en el área de salud mental, entre otros actores.

Uno de las primeras cuestiones que se debatió fue si era, o no, necesario reformar la LC para habilitar estas innovaciones. En las próximas líneas procuraremos presentar los aspectos críticos y las respuestas encontradas para avanzar en modelos institucionales innovadores que puedan dar respuesta a los distintos desafíos identificados en II., aceptando la hipótesis de que ley de cooperativas no se modificará en un mediano plazo.

\subsection{Desarrollo local y asociado colaborador}

El objeto de una cooperativa está definido por el servicio que brinda a sus asociados. El objeto de una cooperativa agropecuaria es comercializar la producción o proveer insumos a sus asociados, el de una cooperativa de consumo proveer de productos de consumo a sus asociados, el de una cooperativa de trabajo, crear trabajo para sus asociados en las condiciones colectivamente acordadas, etc.

Pero, ¿qué pasa cuando los asociados no reciben un servicio específico, sino que se asocian buscando el efecto indirecto que tendrá la cooperativa en beneficio de su comunidad, a través de los salarios que paga, los impuestos que tributa o las cadenas de proveedores que mo-

9 La Confederación Cooperativas de la República Argentina (Cooperar), es una organización nacional de cúpula que integra a 78 entidades, incluyendo federaciones con presencia en todos los sectores y provincias. Organiza el trabajo colaborativo de sus asociadas en Comisiones, una de las cuales es la Comisión de Normativa, abocada a temas legales e impositivos. 
viliza? Esa es la experiencia de las cooperativas que hemos caracterizado como «comunitarias de desarrollo local» en 2.2.

Adecuar los estatutos para que reflejen esta situación, no parece un escollo importante. Basta con aceptar que estas cooperativas tengan como objeto principal «promover el desarrollo local», y que ello las habilite, por ejemplo, a «desarrollar cualquier actividad económica que genere puestos de trabajo, agregue valor y/o complemente las distintas cadenas de valor con presencia en el municipio».

En estas cooperativas los asociados no lo serían en su carácter de trabajadores, consumidores o productores, sino en su carácter de miembros de la comunidad interesados en los beneficios del desarrollo local. Los designaremos provisoriamente como asociados «colaboradores».

¿Podría esta cooperativa retribuir con sus excedentes el capital aportado por sus asociados? La respuesta es no. De acuerdo al art. 42 de la ley cooperativas, el excedente repartible es exclusivamente el que surge de la diferencia entre el precio y el costo del servicio prestado al asociado, y, en estos casos, el asociado no paga por ningún servicio directo.

En la práctica, las que hemos caracterizado como cooperativas comunitarias de desarrollo local, asignan la totalidad de sus excedentes a reservas irrepartibles. Ello no es visualizado como una restricción por quienes las gestionan. Por el contrario, perciben como una ventaja poder contar con excedentes exentos de tributar ganancias (los excedentes repartibles sí pagan ganancias en cabeza de sus asociados ${ }^{10}$ ) y poder asignar estos excedentes a una reserva, que por su carácter de no repartible fortalece el patrimonio de la cooperativa (a diferencia del capital, que eventualmente puede ser retirado por el asociado al momento de desvincularse de la cooperativa ${ }^{11}$ ).

En el caso de cooperativas que, junto con los asociados colaboradores, tengan asociados que sí operan con ella (en su carácter de productores, consumidores o trabajadores) entonces la Asamblea sí podría autorizar el pago de un interés sobre el capital, con las restricciones establecidas el art. 42, inc. 4 de la LC.

De todas maneras, explorando sobre qué herramientas se podrían diseñar para poder retribuir el financiamiento que pudiesen aportar los socios colaboradores en el caso de cooperativas exclusivamente "comunitarias» (es decir, que tienen solo asociados colaboradores, y por lo

10 Con excepción de las cooperativas de consumo.

11 Posibilidad esta habitualmente restringida por los estatutos al 5\% del capital integrado, de acuerdo a lo autorizado por el art. 31 de la LC. 
tanto no generan excedentes repartibles), se analizó la hipótesis «títulos de financiamiento solidario».

En principio, cualquier persona puede realizar un préstamo a la cooperativa, asumiendo el carácter de acreedor. Si a ese préstamo lo retribuimos con un interés limitado, y buscamos que el asociado asuma riesgos parecidos a los de un propietario, sería algo bastante parecido a un aporte de capital.

Con esa idea, es que se discutieron algunos modelos de estatuto que habilitaran la emisión de "Títulos de Financiamiento Solidario», con los siguientes criterios:

i. Tasa de interés máxima equivalente a la establecida para el interés al capital (art. 42, inc. 4 de la LC).

ii. Amortización sujeta a la suscripción de títulos por un valor equivalente (es decir, el monto principal se devuelve sólo si alguien suscribe títulos por un valor al menos igual a los vencimientos).

iii. El pago de interés podrá posponerse por decisión unilateral de Consejo de Administración justificada por poner en riesgo la situación patrimonial o por falta liquidez. Esta decisión podrá ser apelada en Asamblea.

iv. En el caso anterior, la cooperativa deberá reconocer la tasa de interés de la emisión, pero no pagará interés sobre los intereses adeudados, a los efectos de evitar que la aplicación de interés sobre interés provoque un endeudamiento que no sea financieramente sostenible.

El punto ii) tiene que ver con la posibilidad de asignar el valor de estos títulos al patrimonio neto (no al pasivo), a partir del antecedente de los Títulos de Cooperativos de Capitalización (que son también títulos de deuda, pero retribuidos con los excedentes repartibles) y que de acuerdo a la Resolución Técnica 24 de la Federación de Argentina de Consejos Profesionales de Ciencias Económicas — de carácter obligatorio para las cooperativas por Res. 247/2009 del Inaes- pueden asignarse al patrimonio neto de cumplirse lo propuesto en nuestro inciso ii).

Por supuesto, cabría preguntarse quién estaría dispuesto a suscribir estos títulos solidarios. La respuesta es similar a la que daríamos respecto a la suscripción de acciones de las cooperativas: estamos suponiendo la existencia de relaciones de reciprocidad propias de este tipo de entidades. El asociado estaría dispuesto a recibir una retribución menor a la que podría recibir en otras alternativas de inversión por el impacto que ello supone para su comunidad. 


\subsection{Secciones de trabajo}

Si bien no hay restricciones legales para la autorización de cooperativas de objetos múltiples, la política histórica de la autoridad de aplicación en Argentina ha sido no autorizar cooperativas de trabajo que incluyan otro objeto. Por ejemplo, no se han autorizado en las últimas décadas cooperativas de trabajo y consumo, esto es cooperativas que tengan asociados interesados en acceder al servicio de provisión de consumo y asociados interesados en acceder a un puesto de trabajo en la misma cooperativa ${ }^{12}$. En otras palabras, la política ha sido que no puede haber una sección de trabajo en una cooperativa que no sea de trabajo.

Esta restricción se explica por la compleja relación existente entre las cooperativas de trabajo y las instituciones del derecho laboral. Sin bien para la doctrina cooperativa la relación entre el trabajador asociado y su cooperativa es un acto cooperativo, en los términos definidos en el art. 4 de la LC, ello no ha evitado conflictos permanentes con el derecho laboral (aún hoy, cuando son de trabajo aproximadamente la mitad de las cooperativas con matrícula vigente). No es objeto de estas líneas profundizar el tema, pero sí dar cuenta de que, innovar en este sentido, exige enfrentar problemas no resueltos en el cooperativismo de trabajo.

Lo significativo, es que la autoridad de aplicación acaba de aprobar una resolución que rompe con su política histórica.

Por Resolución 525/2021 aprobó un modelo de estatuto de «Cooperativa de Trabajo y Provisión de Servicios para Productores», donde los asociados son productores rurales (artículo 9 del modelo de estatuto). Allí se establece que la cooperativa deberá contar como mínimo con una sección provisión de servicios y otra de trabajo (artículo 7) y que el retorno se distribuirá «en proporción al trabajo efectivamente prestado por cada uno o en proporción a las operaciones realizadas y/o servicios utilizados por cada asociado, de conformidad al criterio de distribución aprobado por la asamblea para cada ejercicio social» (art. 26).

No fue una decisión sencilla. Requirió un intenso debate con funcionarios de programas de gobierno vinculados al desarrollo rural y con organizaciones de pequeños productores. Para hacerlo el Inaes tomó recaudos que buscan evitar que estas cooperativas sean usadas para la elusión de las normas laborales: a) al menos dos tercios de sus asocia-

12 Ha habido asiladas excepciones a esto, pero donde el asociado consumidor debía ser además trabajador, y la sección consumo no podía operar con no asociados. 
dos deben estar inscriptos en el Registro Nacional de Agricultura Familiar (Ley 27.118) y b) su constitución debe haber sido promovida desde la Secretaría de Agricultura Familiar, Campesina e Indígena.

Habiendo aceptado el Inaes esta posibilidad, que en este caso está orientada a resolver parte de los problemas que hemos descripto en 2.4, la pregunta a responder es cómo minimizar los riesgos del fraude laboral en el resto de las situaciones que hemos analizado. Por ejemplo, en los casos de plataformas cooperativas donde los repartidores operen en una sección de trabajo, o de recuperación de empresas cuando haya otros actores además de los trabajadores.

Una alternativa es exigir que estas cooperativas cuenten con un reglamento que establezca las condiciones de trabajo que debe garantizar a sus asociados trabajadores. El art. 13 de la LC establece que los reglamentos deben ser aprobados por la autoridad de aplicación antes de entrar en vigencia. Es decir, el reglamento, que obligatoriamente deberían tener estas cooperativas para establecer las condiciones de trabajo, debería ser aprobado por el Inaes. La salida que proponemos al problema planteado es consensuar un reglamento que proteja los derechos laborales de los trabajadores asociados, pero dentro de la flexibilidad que supone el carácter asociativo de esta relación laboral.

Si este criterio fuese aceptado no sólo por el Inaes, sino también por el Ministerio de Trabajo, se lograría la protección de los derechos de los trabajadores asociados en cooperativas de múltiples partes interesadas, y al mismo tiempo se minimizarían los conflictos con las instituciones del derecho laboral ${ }^{13}$.

\subsection{Distritos según tipo de vínculo asociativo}

El art. 19 de la LC establece que cuando el Estado nacional, provincial o municipal, entes descentralizados o empresas del Estado se asocien a una cooperativa «pueden convenir la participación que les corresponderá en la administración y fiscalización de sus actividades en cuanto fuera coadyuvante a los fines perseguidos y siempre que tales convenios no restrinjan la autonomía de la cooperativa».

13 Este, en realidad, puede ser un camino para resolver las tensiones de todas las cooperativas de trabajo con las instituciones del derecho laboral. Por ello, Cooperar propone que se incorpore un capítulo de cooperativas de trabajo en la LC, donde, entre otros temas, se establezca la obligatoriedad de contar con un reglamento de condiciones de trabajo (Cooperar, 2019: 75). 
En el marco de dicho artículo existen estatutos de cooperativas de servicios públicos que establecen que, en su Consejo de Administración, siempre debe haber un representante del Gobierno Municipal de la localidad donde brinda el servicio.

Es un antecedente valioso, en tanto reconoce derechos especiales a un asociado, siempre y cuando no se restrinja la autonomía de la cooperativa. Esto habilita la posibilidad de reconocer una participación especial del Municipio en el consejo de administración de la cooperativa, por ejemplo, cuando se busque organizar una cooperativa comunitaria para crear trabajo, o para recuperar una empresa, en tanto el municipio es representante de la comunidad interesada en el desarrollo del emprendimiento. Incluso podría reconocerse esta posibilidad a otras instituciones de carácter comunitario, no estatales, como por ejemplo las cooperativas de servicios públicos.

Por otro lado, en el art. 50 de la LC, se establece la posibilidad de distritos para el ejercicio de los derechos políticos de los asociados cuando su número es grande, o cuando se encuentran alejados del domicilio de la cooperativa. Esta división de los asociados según el distrito de carácter territorial al que pertenecen, previsto por la LC, permite pensar, por analogía, en la posibilidad de dividir los asociados en distritos según el tipo de vínculo que tienen con la cooperativa (consumidores, usuarios, trabajadores, productores, colaboradores, etc.).

Por ejemplo, podría pensarse una Plataforma Cooperativa, con asociados del distrito trabajo (repartidores), del distrito usuario (pymes y comercios adheridos) y del distrito colaboradores (instituciones locales que acompañan la iniciativa). Todos ellos participarían en la Asamblea con un voto, pero esta Asamblea, por estatuto, estaría obligada a elegir a los miembros del Consejo de Administración con un número fijo de integrantes pertenecientes a cada distrito (no necesariamente iguales) ${ }^{14}$.

A partir de estos dos criterios, a) la posibilidad de reconocer una participación especial en el consejo de determinadas instituciones, y b) la posibilidad de dividir el padrón de asociados en distritos de acuerdo al vínculo que tienen con la cooperativa, entendemos que es posible resolver un amplio espectro de alternativas de gobernanza que reflejen acuerdos colaborativos entre distintos actores.

${ }^{14}$ En el caso de que el número de asociados lo requiera, los asociados de cada distrito podrían elegir delegados que participarían a la Asamblea. Limitar el número de delegados para que no haya una representación desequilibrada en la Asamblea (por ejemplo entre usuarios y trabajadores en una cooperativas que reconociera en su gobernanza estos dos tipos de vínculos) es una posibilidad más discutible si no se reforma la LC. 
Por ejemplo, para el caso de cooperativas de inserción socio laboral de usuarios de servicios de salud mental, durante 2020 se trabajó en la posibilidad de reformar sus estatutos para que los asociados se dividan en dos distritos (trabajadores y colaboradores), a los que se sumaría la participación del Servicio Público de Salud Mental en el Consejo de Administración.

Al reconocimiento de distritos por tipo de vínculo asociativo, puede sumarse el reconocimiento de distritos por organización de base a la que pertenece. Expliquemos este punto.

Supongamos que se está pensando la organización de una cooperativa de abastecimiento de productos de la economía social, como los casos que presentamos en 2.5. Los actores de este proceso son organizaciones de proveedores y organizaciones de consumidores. Una posibilidad es constituir una cooperativa que asocie a las organizaciones preexistentes ${ }^{15}$. Sin embargo, ello puede ser impracticable si las organizaciones no pueden realizar operaciones comerciales (por ejemplo, asociaciones civiles), o si este tipo de integración no resulta conveniente por sus efectos tributarios y/o si resulta engorrosa por exigir la participación de más de una administración descentralizada en una misma operación de abastecimiento. Aquí se presenta un dilema, dado que las organizaciones preexistentes pueden tener resistencia a ser diluidas en el marco de la nueva cooperativa.

Para salvar esto, la cooperativa puede reconocer a cada organización preexistente como un distrito, a los efectos del ejercicio de los derechos políticos de sus asociados (ya sea para garantizar una distribución equitativa de los miembros del Consejo de Administración según la organización a la que pertenecen, o para designar a los delegados que participarán de la Asamblea de Delegados).

Esto tiene antecedentes en Argentina. Hay cooperativas aseguradoras, que cubren riesgos agrícolas, donde se reconocen como distritos, a los efectos de elegir sus delegados, a las cooperativas agropecuarias a las que también están asociados sus miembros. También existen cooperativas agropecuarias, donde se exige que sus asociados formen parte de algún grupo de transferencia de tecnología, y se les reconoce

15 Como bien aclara la Res. 478/2020 de Cooperativas de Múltiples partes interesadas, las cooperativas de primer grado pueden tener asociados de distintas figuras jurídicas sin limitaciones, y por lo tanto servir como herramienta de integración de distintas organizaciones. También existe la posibilidad de organizar una cooperativa de grado superior (art. 85 LC), que tendría la ventaja de poder reconocer voto múltiple de acuerdo, por ejemplo, a la cantidad de asociados, pero la desventaja de que la mayoría de las asociadas deben ser cooperativas (Res. 597/95). 
a estos una participación equitativa en el Consejo de Administración (Basañes, Amantini, 2017: 61).

\section{Conclusión}

El cooperativismo argentino enfrenta el desafío de desarrollar modelos institucionales innovadores para viabilizar estratégicas asociativas en el campo de la economía digital, la inserción socio-laboral de personas con desventajas, la recuperación de empresas en crisis y otros estrechamente vinculados al cumplimiento de los objetivos de desarrollo sostenible y a superar las fragilidades sociales que ha puesto en evidencia el Covid-19.

Ante esto, en el marco de la flexibilidad y diversidad que ha caracterizado la experiencia cooperativa en este país, y como continuidad de la decisión política expresada por el Directorio del Inaes de posibilitar la constitución de cooperativas de múltiples partes interesadas, resulta factible avanzar en la aprobación de estatutos y reglamentos innovadores a partir del reconocimiento del desarrollo local como objeto que justifique la figura del asociado colaborador, la autorización de secciones de trabajo en cooperativas que no sean exclusivamente de trabajo (siguiendo el antecedente de la resolución 525/2021) y el reconocimiento de distritos de asociados según tipo de vínculo asociativo.

Esto debería facilitar un proceso de aprendizaje participativo, liderado por los emprendedores sociales que están buscando nuevos caminos para responder desde la democracia económica y la ayuda mutua a los nuevos desafíos globales, todo ello acompañado por una inteligente adaptación de las normas y prácticas de la autoridad de aplicación.

\section{Referencias}

BASAÑES, C.: Integración Cooperativa: Reflexiones a propósito del anteproyecto de Ley Federal de Cooperativas y Mutuales. Documento de Discusión. Confederación Cooperativa de la República Argentina. Buenos Aires, Argentina, 2015.

BASAÑES, C.; AMANTINI, A.: Innovaciones Institucionales en cooperativas agropecuarias en la República Argentina. Instituto Interamericano de Cooperación para la Agricultura IICA Argentina, Buenos Aires, 2017. http://repositorio. iica.int/bitstream/handle/11324/6464/BVE18029654e.pdf;jsessionid=E3DA BDA54664397BAF13641048A9D595? sequence $=1$

BRAGULAT, J. (comp.): Las cooperativas sociales en la prestación de servicios asistenciales Análisis de experiencias y de su potencial desarrollo en América 
Latina. EDUNTREF. Editorial de la Universidad Nacional de Tres de Febrero. Buenos Aires, Argentina, 2020.

COOPERAR: Aportes para un Plan Nacional de Desarrollo. Documento de la Confederación Cooperativa de la República Argentina, 2019. https://www.cooperar.coop/wp-content/uploads/2019/10/Aportes-para-un-Plan-Nacionalde-Desarrollo-COOPERAR-V2.pdf

ERRECALDE, S. (comp.): La economía popular ante la crisis: por la defensa de los derechos y hacia una economía social y ambientalmente sustentable. Varios autores. Cuadernos de la Economía Social y Solidaria. Universidad Nacional de Quilmes. Berazategui, Argentina, 2020. http://observatorioess. org.ar/coness/?fbclid=IWAR1XDMAW9HNIgVLI18TMFnHu4tkKmPsLqTgzXSThBMeaOnxRSo9EPWh-NA

Guarco, A.: Principios Cooperativos en Acción. Frente a los desafíos de la agenda global. InterCoop Coop. Ltda., Buenos Aires, Argentina, 2020.

JuRAdo, E.; Gallo, M.: Economía social y solidaria en Río Negro y Mendoza. Políticas públicas, sujetos y espacialidades en debate. Revista Idelcoop, n. ${ }^{\circ} 221$. Buenos Aires, Argentina, 2017.

MAYO, E.: ¿Democracia digital? Opciones de la Alianza Cooperativa Internacional para avanzar en las cooperativas de plataforma. Documento de Discusión de la Alianza Cooperativa Internacional. 2019. https://www.uk.coop/ sites/default/files/2020-11/annexo_1_-_documento_de_discusion_-_es.pdf

Obschatko, E; Basañes, C.; Martini, G.: Las Cooperativas Agropecuarias en la República Argentina. Diagnóstico y Propuestas. Instituto Interamericano de Cooperación para la Agricultura IICA Argentina, Ministerio de Agricultura Ganadería y Pesca, Buenos Aires, Argentina, 2011. http://fidamercosur.org/ claeh/images/BIBLIOTECA/FasellI_2012/Noviembre/SeminarioChile/MAGyPIICA-Las\%20cooperativas\%20agropecuarias\%20en\%20la\%20RA.pdf

SuARez AreA, P.: Cooperativas de servicios públicos. Hacia una perspectiva endógena y local del desarrollo. Revista Realidad Económica. Vol. 47. Núm. 318. Buenos Aires, Argentina, 2018. https://ojs.iade.org.ar/index. php/re/article/view/30

VANNINI, P.: Capitalismo cognitivo y plataformas, una mirada desde la economía social. Revista Idelcoop, n. ${ }^{\circ} 228$. Buenos Aires, Argentina, 2019. 


\section{Derechos de autor}

La revista Deusto Estudios Cooperativos es una revista de acceso abierto lo que significa que es de libre acceso en su integridad inmediatamente después de la publicación de cada número. Se permite su lectura, la búsqueda, descarga, distribución y reutilización legal en cualquier tipo de soporte sólo para fines no comerciales y según lo previsto por la ley; sin la previa autorización de la Editorial (Universidad de Deusto) o el autor, siempre que la obra original sea debidamente citada (número, año, páginas y DOI si procede) y cualquier cambio en el original esté claramente indicado.

\section{Copyright}

The Deusto Journal of Cooperative Studies is an Open Access journal which means that it is free for full and immediate access, reading, search, download, distribution, and lawful reuse in any medium only for non-commercial purposes, without prior permission from the Publisher or the author; provided the original work is properly cited and any changes to the original are clearly indicated. 